\title{
Gap functions and error bounds for vector inverse mixed quasi-variational inequality problems
}

\author{
Zhong-bao Wang ${ }^{1,2^{*}}$, Zhang-you Chen ${ }^{1}$ and Zhe Chen ${ }^{3}$
}

\author{
"Correspondence: \\ zhongbaowang@hotmail.com \\ 'Department of Mathematics, \\ Southwest Jiaotong University, \\ Chengdu, China \\ ${ }^{2}$ School of Mathematical Sciences, \\ University of Electronic Science and \\ Technology of China, Chengdu, \\ China \\ Full list of author information is \\ available at the end of the article
}

\begin{abstract}
This paper is devoted to investigating a vector inverse mixed quasi-variational inequality (VIMQVI). Our aim is to obtain error bounds for VIMQVI in terms of different gap functions, i.e., the residual gap function, the regularized gap function, and the $D$-gap function. These bounds provide effective estimated distances between an arbitrary feasible point and the solution set of VIMQVI. The approach exploited in this paper is based on the generalized $f$-projection operator due to Wu and Huang. Our results cover and extend similar results for these problems.
\end{abstract}

MSC: 49J53; 49J53

Keywords: Vector inverse mixed quasi-variational inequality; Gap function; Error bounds; Generalized $f$-projection operator

\section{Introduction}

Variational inequalities (VI) and quasi-variational inequalities (QVI) have many applications in different fields such as economics, management, and engineering. An important and useful generalization of VI is called the mixed variational inequality (MVI). Some results and applications related to MVI have been studied by many authors (see, for example, $[1-3])$.

Recently, He et al. [4,5] introduced inverse variational inequalities (IVI). As pointed in [6], many applications of IVI can be founded in various areas such as market equilibrium problems in economics and telecommunication networks. Li et al. [7] introduced a new inverse mixed variational inequality (IMVI) in the setting of Hilbert spaces, which includes IVI as a special case. An example concerned with a simple traffic network equilibrium control problem was given to illustrate the applicability of IMVI.

In 1980, vector variational inequalities (VVI) were initiated in the setting of the finitedimensional Euclidean space, see [8]. This is a generalization of scalar variational inequalities to the vector case by virtue of multi-criterion consideration. So far, vector variational inequalities has been applied to optimization, optimal control, operations research, economics equilibrium, and free boundary value problems. In the past decades, existence, stability, sensitivity, optimality conditions, and differentiability for solutions of VVI and their various extensions have been extensively studied, see [8-19] and the references therein.

(c) The Author(s) 2019. This article is distributed under the terms of the Creative Commons Attribution 4.0 International License (http://creativecommons.org/licenses/by/4.0/), which permits unrestricted use, distribution, and reproduction in any medium, provided you give appropriate credit to the original author(s) and the source, provide a link to the Creative Commons license, and indicate if changes were made. 
The concept of gap function was first introduced for the study of optimization problems and subsequently applied to VI, QVI, and VVI. Gap functions play an important part in developing iterative algorithms but more importantly for analyzing their convergence properties and obtaining useful stopping rules for iterative algorithms. We refer readers to $[15,20-29]$ for surveys. Error bounds are very important and useful as they provide a measure of the distance between a solution set and an arbitrary feasible point. A comprehensive survey of theory and rich applications about error bounds can be found in [30]. Solodov [26] constructed some merit functions associated with a generalized MVI (which was defined on the whole space) and used those functions to obtain error bounds for MVI. Recently, Aussel et al. [31] introduced a new inverse quasi-variational inequality (IQVI), obtained local (global) error bounds for IQVI in terms of some gap functions to illustrate the applicability of IQVI, and gave an example about road pricing problems. Sun and Chai [32] introduced the regularized gap functions for the generalized vector variational inequalities (GVVI) and obtained the error bounds for GVVI in terms of the regularized gap functions. Charitha et al. [33] studied several gap functions for Stampacchia and Minty-type VVI and developed error bounds for the VVI with strongly monotone data in terms of the several gap functions. The generalized $f$-projection operators introduced by Wu and Huang [34] was exploited to deal with MVI, see, for example, [7, 34-37]. Very recently, by using the generalized $f$-projection operator, Li and Li [37] investigated a constrained mixed set-valued variational inequality (MSVI) in Hilbert spaces, and proposed four merit functions for the constrained MSVI and obtained error bounds by these functions. A natural question is whether one can give some model to unify IVI, IMVI, IQVI, VVI, and GVVI, and furthermore study their gap functions and the corresponding error bounds or not.

In this paper, we introduce a vector inverse mixed quasi-variational inequality (VIMQVI), which includes IVI, IMVI, IQVI, VVI, and GVVI as special cases. We also propose three gap functions for the VIMQVI, i.e., the residual gap function, the regularized gap function, and the $D$-gap function, and obtain error bounds for VIMQVI under strong monotonicity and Lipschitz continuity of underlying mappings by using these gap functions. Our basic tool is the generalized $f$-projection operator due to Wu and Huang, which is more general than the well-known proximal mapping exploited in [26].

\section{Preliminaries}

Throughout this paper, let the set of nonnegative real numbers be denoted by $R_{+}$, the origins of all finite dimensional spaces be denoted by 0 , and the norms and the inner products of all finite dimensional spaces be denoted by $\|\cdot\|$ and $\langle\cdot, \cdot\rangle$, respectively. Furthermore, let $K: R^{n} \rightarrow 2^{R^{n}}$ be a set-valued mapping with nonempty closed convex values, $F_{i}: R^{n} \rightarrow R^{n}$ $(i=1,2, \ldots, m)$ be single-valued mappings, $h: R^{n} \rightarrow R^{n}$ be a single-valued mapping, and $f_{i}: R^{n} \rightarrow R(i=1,2, \ldots, m)$ be real-valued convex functions. For abbreviation, we put

$$
T:=\left(f_{1}, f_{2}, \ldots, f_{m}\right), \quad F:=\left(F_{1}, F_{2}, \ldots, F_{m}\right),
$$

and for any $x, v \in R^{n}$,

$$
\langle F(x), v\rangle:=\left(\left\langle F_{1}(x), v\right\rangle,\left\langle F_{2}(x), v\right\rangle, \ldots,\left\langle F_{n}(x), v\right\rangle\right) .
$$


In this paper, we consider the following vector inverse mixed quasi-variational inequality (in short, VIMQVI): find $\bar{x} \in K(\bar{x})$ such that

$$
\langle F(\bar{x}), y-h(\bar{x})\rangle+T(y)-T(h(\bar{x})) \notin-\operatorname{int} R_{+}^{m}, \quad \forall y \in K(\bar{x}) .
$$

The solution set of VIMQVI is denoted by sol(VIMQVI).

If $C \subset R^{n}$ is a nonempty closed and convex subset, $h(x)=x$ and $K(x)=C$ for all $x \in R^{n}$, then VIMQVI collapses to the following GVVI: find $\bar{x} \in C$ such that

$$
\langle F(\bar{x}), y-x\rangle+T(y)-T(x) \notin-\operatorname{int} R_{+}^{m}, \quad \forall y \in C,
$$

which is considered and studied by [32].

If $T(x)=0$ for all $x \in R^{n}$, then GVVI reduces to VVI introduced and studied by [11, 12, 33].

Obviously, for $m=1$, VIMQVI collapses to the following inverse mixed quasi-variational inequality (IMQVI): find $\bar{x} \in K(\bar{x})$ such that

$$
\left\langle F_{1}(\bar{x}), y-h(\bar{x})\right\rangle+f_{1}(y)-f_{1}(h(\bar{x})) \geq 0, \quad \forall y \in K(\bar{x}),
$$

which was introduced and studied by [36].

If $f_{1}(x)=0$ for all $x \in R^{n}$, then IMQVI collapses to the following IQVI:

$$
\left\langle F_{1}(\bar{x}), y-h(\bar{x})\right| \geq 0, \quad \forall y \in K(\bar{x}) .
$$

This problem was considered and studied by Aussel et al. [31], who pointed out that the discipline of IQVI is still not fully explored and much is desired to be done. Clearly, IQVI includes the classes of general quasi-variational inequalities and variational inequalities as special cases.

If $C \subset R^{n}$ is a nonempty closed and convex subset and $K(x)=C$ for all $x \in R^{n}$, then IMQVI collapses to the following MVI: find $\bar{x} \in C$ such that

$$
\left\langle F_{1}(\bar{x}), y-h(\bar{x})\right\rangle+f_{1}(y)-f_{1}(h(\bar{x})) \geq 0, \quad \forall y \in C .
$$

When $C=R^{n}$, MVI was investigated by Solodov [26]; when $F_{1}(x)=x, \forall x \in R^{n}$, MVI becomes IMVI which was introduced and studied by [7].

For $i=1,2, \ldots, m$, we denote the inverse mixed quasi-variational inequality associated with $F_{i}, h, K$, and $f_{i}$ as (IMQVI) ${ }^{i}$. The solution sets of (IMQVI) ${ }^{i}$ are denoted by sol (IMQVI) ${ }^{i}$.

In this paper, we intend to investigate several scalar-valued gap functions and error bounds for VIMQVI. In order to do so, we shall recall some notations and definitions, which will be used in the sequel.

Definition 2.1 [31] Let $G: R^{n} \rightarrow R^{n}$ and $g: R^{n} \rightarrow R^{n}$ be two maps.

(i) $(G, g)$ is said to be a strongly monotone couple with modulus $\mu$ if there exists a constant $\mu>0$ such that

$$
\langle G(y)-G(x), g(y)-g(x)\rangle \geq \mu\|y-x\|^{2}, \quad \forall x, y \in R^{n}
$$


(ii) $g$ is said to be $L$-Lipschitz continuous on $R^{n}$ if there exists a constant $L>0$ such that

$$
\|g(x)-g(y)\| \leq L\|x-y\|, \quad \forall x, y \in R^{n} .
$$

For any fixed $\rho>0$, let $G: R^{n} \times \tilde{K} \rightarrow(-\infty,+\infty]$ be a function defined as follows:

$$
G(\varphi, x)=\|x\|^{2}-2\langle\varphi, x\rangle+\|\varphi\|^{2}+2 \rho f(x), \quad \forall \varphi \in R^{n}, \forall x \in \tilde{K},
$$

where $\tilde{K} \subset R^{n}$ is a nonempty closed and convex subset, and $f: R^{n} \rightarrow R$ is convex.

Definition $2.2([34])$ We say that $\Pi_{\tilde{K}}^{f}: R^{n} \rightarrow 2^{\tilde{K}}$ is a generalized $f$-projection operator if

$$
\Pi_{\tilde{K}}^{f} \varphi=\left\{u \in \tilde{K}: G(\varphi, u)=\inf _{y \in \tilde{K}} G(\varphi, y)\right\}, \quad \forall \varphi \in R^{n}
$$

If $f(x)=0$ for all $x \in \tilde{K}$, then the generalized $f$-projection operator $\Pi_{\tilde{K}}^{f}$ is equivalent to the following metric projection operator:

$$
P_{\tilde{K}}(\varphi)=\left\{u \in \tilde{K}:\|u-\varphi\|=\inf _{y \in \tilde{K}}\|y-\varphi\|\right\}, \quad \forall \varphi \in R^{n} .
$$

Lemma $2.1([7,34])$ The following statements hold:

(i) For any given $\varphi \in R^{n}, \Pi_{\tilde{K}}^{f} \varphi$ is nonempty and single-valued;

(ii) For any given $\varphi \in R^{n}, x=\Pi_{\tilde{K}}^{f} \varphi$ if and only if

$$
\langle x-\varphi, y-x\rangle+\rho f(y)-\rho f(x) \geq 0, \quad \forall y \in \tilde{K}
$$

(iii) $\Pi_{\tilde{K}}^{f}: R^{n} \rightarrow K$ is nonexpansive, that is, $\left\|\Pi_{\tilde{K}}^{f} x-\Pi_{\tilde{K}}^{f} y\right\| \leq\|x-y\|$ for all $x, y \in R^{n}$.

Lemma 2.2 ([36]) Let $m$ be a positive number, $B \subset R^{n}$ be a nonempty subset such that $\|v\| \leq m$ for all $v \in B$. Let $K: R^{n} \rightarrow 2^{R^{n}}$ be a set-valued mapping such that, for each $x \in R^{n}$, $K(x)$ is a closed convex set, and let $f: R^{n} \rightarrow R$ be a convex function on $R^{n}$. Assume that

(i) there exists a constant $\gamma>0$ such that $H(K(x), K(y)) \leq \gamma\|x-y\|, x, y \in R^{n}$;

(ii) $0 \in \bigcap_{u \in R^{n}} K(u)$;

(iii) $f$ is l-Lipschitz continuous on $R^{n}$. Then there exists a constant $k=\sqrt{6 \gamma(m+\rho l)}$ such that

$$
\left\|\Pi_{K(x)}^{f} z-\Pi_{K(x)}^{f} z\right\| \leq k\|x-y\|, \quad \forall x, y \in R^{n}, z \in B
$$

Definition 2.3 A function $r: R^{n} \rightarrow R$ is said to be a gap function for a VIMQVI on a set $\tilde{S} \subset R^{n}$ if it satisfies the following properties:

(i) $r(x) \geq 0$ for any $x \in \tilde{S}$;

(ii) $r(\bar{x})=0, \bar{x} \in \tilde{S}$ if and only if $\bar{x}$ is a solution of VIMQVI.

The gap functions play an important part in developing iterative algorithms for solving VIMQVI but more importantly for analyzing their convergence properties and obtaining useful stopping rules for iterative algorithms. This motivates us to study and analyze different gap functions for VIMQVI. 


\section{Residual gap functions}

In this section, we shall give the residual gap function for VIMQVI and prove error bounds related to the residual gap function. We define the residual gap function for VIMQVI as follows:

$$
r_{\rho}(x):=\min _{1 \leq i \leq m}\left\{\left\|h(x)-\Pi_{K(x)}^{f_{i}}\left[h(x)-\rho F_{i}(x)\right]\right\|\right\}, \quad x \in R^{n}, \rho>0 .
$$

Theorem 3.1 Suppose that $F_{i}: R^{n} \rightarrow R^{n}(i=1,2, \ldots, m)$ are single-valued mappings, then for any $\rho>0, r_{\rho}(x)$ is a gap function for VIMQVI on $R^{n}$.

Proof It is clear that $r_{\rho}(x) \geq 0$ for any $x \in R^{n}$. On the other hand, if $r_{\rho}(\bar{x})=0$, then there exists $0 \leq i_{0} \leq m$ such that

$$
h(\bar{x})=\Pi_{K(\bar{x})}^{f_{i_{0}}}\left[h(\bar{x})-\rho F_{i_{0}}(\bar{x})\right] .
$$

Lemma 2.1 implies that

$$
\left\langle h(\bar{x})-\left[h(\bar{x})-\rho F_{i_{0}}(\bar{x})\right], y-h(\bar{x})\right\rangle+\rho f(y)-\rho f(h(\bar{x})) \geq 0, \quad \forall y \in K(\bar{x}),
$$

and so

$$
\left\langle F_{i_{0}}(\bar{x}), y-h(\bar{x})\right\rangle+f(y)-f(h(\bar{x})) \geq 0, \quad \forall y \in K(\bar{x}) .
$$

This means that

$$
\langle F(\bar{x}), y-h(\bar{x})\rangle+f(y)-f(h(\bar{x})) \notin-\operatorname{int} R_{+}^{m}, \quad \forall y \in K(\bar{x}) .
$$

Thus, $\bar{x}$ is a solution of VIMQVI.

Conversely, if $\bar{x}$ is a solution of VIMQVI, there exists $1 \leq i_{0} \leq m$ such that

$$
\left\langle F_{i_{0}}(\bar{x}), y-h(\bar{x})\right\rangle+f_{i_{0}}(y)-f_{i_{0}}(h(\bar{x})) \geq 0, \quad \forall y \in K(\bar{x}) .
$$

By Lemma 2.1, we have

$$
h(\bar{x})=\Pi_{K(\bar{x})}^{f_{i_{0}}}\left[h(\bar{x})-\rho F_{i_{0}}(\bar{x})\right] .
$$

This means that

$$
r_{\rho}(\bar{x})=\min _{1 \leq i \leq m}\left\{\left\|h(\bar{x})-\Pi_{K(\bar{x})}^{f_{i}}\left[h(\bar{x})-\rho F_{i}(\bar{x})\right]\right\|\right\}=0 .
$$

This completes the proof.

Next we will give the error bound for VIMQVI in terms of the residual gap function $r_{\rho}$.

Theorem 3.2 Let $F_{i}: R^{n} \rightarrow R^{n}(i=1,2, \ldots, m)$ be $L_{i}$-Lipschitz continuous, $h: R^{n} \rightarrow R^{n}$ be l-Lipschitz continuous, and for $i=1,2, \ldots, m,\left(F_{i}, h\right)$ be strongly monotone couples with 
modulus $\mu_{i}$. Let $\bigcap_{i=1}^{m} \operatorname{sol}(\mathrm{IMQVI})^{i} \neq \emptyset$. Assume that there exists $k_{i} \in\left(0, \frac{\mu_{i}}{L_{i}}\right)$ such that

$$
\left\|\Pi_{K(x)}^{f_{i}} z-\Pi_{K(y)}^{f_{i}} z\right\| \leq k_{i}\|x-y\|, \quad \forall x, y \in R^{n}, z \in\left\{v \mid v=h(x)-\rho F_{i}(x)\right\} .
$$

Then, for any $x \in R^{n}$ and $\rho>\frac{k_{i} l}{\mu_{i}-k_{i} L_{i}}$,

$$
d(x, \operatorname{Sol}(\text { VIMQVI })) \leq \frac{\rho L_{i}+l}{\rho \mu_{i}-\rho k_{i} L_{i}-k_{i} l} r_{\rho}(x),
$$

where $d(x, \operatorname{Sol}(\mathrm{VIMQVI}))=\inf _{\bar{x} \in \operatorname{Sol}(\mathrm{VIMQVI})}\|x-\bar{x}\|$ denotes the distance between the point $x$ and the set Sol(VIMQVI).

Proof Because $\bigcap_{i=1}^{m} \operatorname{sol}(\mathrm{IMQVI})^{i} \neq \emptyset$, we assume that $\bar{x} \in K(\bar{x})$ is a common solution of (IMQVI) $^{i}, i=1, \ldots, m$, and thus for any $i \in\{1, \ldots, m\}$, we have

$$
\left\langle F_{i}(\bar{x}), y-h(\bar{x})\right\rangle+f_{i}(y)-f_{i}(h(\bar{x})) \geq 0, \quad \forall y \in K(\bar{x}) .
$$

By definition of $\Pi_{K(\bar{x})}^{f_{i}}\left[h(x)-\rho F_{i}(x)\right]$, Lemma 2.1 implies that

$$
\begin{aligned}
& \left\langle\Pi_{K(\bar{x})}^{f_{i}}\left[h(x)-\rho F_{i}(x)\right]-\left(h(x)-\rho F_{i}(x)\right), y-\Pi_{K(\bar{x})}^{f_{i}}\left[h(x)-\rho F_{i}(x)\right]\right\rangle \\
& \quad+\rho f_{i}(y)-\rho f_{i}\left(\Pi_{K(\bar{x})}^{f_{i}}\left[h(x)-\rho F_{i}(x)\right]\right) \geq 0, \quad \forall y \in K(\bar{x}) .
\end{aligned}
$$

Since $\bar{x} \in \bigcap_{i=1}^{m} \operatorname{sol}(\mathrm{IMQVI})^{i}, h(\bar{x}) \in K(\bar{x})$. Replacing $y$ by $h(\bar{x})$ in $(5)$, we get

$$
\begin{aligned}
& \left\langle\Pi_{K(\bar{x})}^{f_{i}}\left[h(x)-\rho F_{i}(x)\right]-\left(h(x)-\rho F_{i}(x)\right), h(\bar{x})-\Pi_{K(\bar{x})}^{f_{i}}\left[h(x)-\rho F_{i}(x)\right]\right\rangle \\
& \quad+\rho f_{i}(h(\bar{x}))-\rho f_{i}\left(\Pi_{K(\bar{x})}^{f_{i}}\left[h(x)-\rho F_{i}(x)\right]\right) \geq 0 .
\end{aligned}
$$

From $\Pi_{K(\bar{x})}^{f_{i}}\left[h(x)-\rho F_{i}(x)\right] \in K(\bar{x})$, by (4), it follows that

$$
\begin{aligned}
& \left\langle\rho F_{i}(\bar{x}), \Pi_{K(\bar{x})}^{f_{i}}\left[h(x)-\rho F_{i}(x)\right]-h(\bar{x})\right\rangle \\
& \quad+\rho f_{i}\left(\Pi_{K(\bar{x})}^{f_{i}}\left[h(x)-\rho F_{i}(x)\right]\right)-\rho f_{i}(h(\bar{x})) \geq 0 .
\end{aligned}
$$

By (6) and (7), we have

$$
\left\langle\rho F_{i}(\bar{x})-\rho F_{i}(x)-\Pi_{K(\bar{x})}^{f_{i}}\left[h(x)-\rho F_{i}(x)\right]+h(x), \Pi_{K(\bar{x})}^{f_{i}}\left[h(x)-\rho F_{i}(x)\right]-h(\bar{x})\right\rangle \geq 0,
$$

which also implies

$$
\begin{aligned}
& \left\langle\rho F_{i}(\bar{x})-\rho F_{i}(x), \Pi_{K(\bar{x})}^{f_{i}}\left[h(x)-\rho F_{i}(x)\right]-h(x)\right\rangle \\
& \quad-\left\langle\rho F_{i}(\bar{x})-\rho F_{i}(x), h(\bar{x})-h(x)\right\rangle \\
& \quad+\left\langle h(x)-\Pi_{K(\bar{x})}^{f_{i}}\left[h(x)-\rho F_{i}(x)\right], \Pi_{K(\bar{x})}^{f_{i}}\left[h(x)-\rho F_{i}(x)\right]-h(x)\right\rangle \\
& \quad+\left\langle h(x)-\Pi_{K(\bar{x})}^{f_{i}}\left[h(x)-\rho F_{i}(x)\right], h(x)-h(\bar{x})\right\rangle \geq 0 .
\end{aligned}
$$


Since, for $i=1,2, \ldots, m,\left(F_{i}, h\right)$ are strongly monotone couples with modulus $\mu_{i}$, we have

$$
\begin{aligned}
& \left\langle\rho F_{i}(\bar{x})-\rho F_{i}(x), \Pi_{K(\bar{x})}^{f_{i}}\left[h(x)-\rho F_{i}(x)\right]-h(x)\right\rangle \\
& \quad-\left\|h(x)-\Pi_{K(\bar{x})}^{f_{i}}\left[h(x)-\rho F_{i}(x)\right]\right\|^{2} \\
& \quad+\left\langle h(x)-\Pi_{K(\bar{x})}^{f_{i}}\left[h(x)-\rho F_{i}(x)\right], h(x)-h(\bar{x})\right\rangle \geq \rho \mu_{i}\|x-\bar{x}\|^{2} .
\end{aligned}
$$

By inserting $\Pi_{K(x)}^{f_{i}}\left[h(x)-\rho F_{i}(x)\right]$ and using the Cauchy-Schwarz inequality along with the triangular inequality, we have

$$
\begin{aligned}
& \left\|\rho F_{i}(\bar{x})-\rho F_{i}(x)\right\| \cdot\left\{\left\|\Pi_{K(\bar{x})}^{f_{i}}\left[h(x)-\rho F_{i}(x)\right]-\Pi_{K(x)}^{f_{i}}\left[h(x)-\rho F_{i}(x)\right]\right\|\right. \\
& \left.\quad+\left\|\Pi_{K(x)}^{f_{i}}\left[h(x)-\rho F_{i}(x)\right]-h(x)\right\|\right\}+\|h(x)-h(\bar{x})\| \\
& \quad \cdot\left\{\left\|h(x)-\Pi_{K(x)}^{f_{i}}\left[h(x)-\rho F_{i}(x)\right]\right\|\right. \\
& \left.\quad+\left\|\Pi_{K(x)}^{f_{i}}\left[h(x)-\rho F_{i}(x)\right]-\Pi_{K(\bar{x})}^{f_{i}}\left[h(x)-\rho F_{i}(x)\right]\right\|\right\} \geq \rho \mu_{i}\|x-\bar{x}\|^{2} .
\end{aligned}
$$

Using the Lipschitz continuity of $F_{i}, h$ and condition (3), we have

$$
\begin{aligned}
& L_{i} \rho\|\bar{x}-x\| \cdot\left(k_{i}\|\bar{x}-x\|+\left\|\Pi_{K(x)}^{f_{i}}\left[h(x)-\rho F_{i}(x)\right]-h(x)\right\|\right) \\
& \quad+l\|x-\bar{x}\| \cdot\left(\left\|h(x)-\Pi_{K(x)}^{f_{i}}\left[h(x)-\rho F_{i}(x)\right]\right\|+k_{i}\|x-\bar{x}\|\right) \geq \rho \mu_{i}\|x-\bar{x}\|^{2} .
\end{aligned}
$$

Hence, for any $x \in R^{n}$ and $i \in\{1,2, \ldots, m\}, \rho>\frac{k_{i} l}{\mu_{i}-k_{i} L_{i}}$ and $\mu_{i}>k_{i} L_{i}$, we have

$$
\|x-\bar{x}\| \leq \frac{\rho L_{i}+l}{\rho \mu_{i}-\rho k_{i} L_{i}-k_{i} l}\left\|h(x)-\Pi_{K(x)}^{f_{i}}\left[h(x)-\rho F_{i}(x)\right]\right\| .
$$

This implies

$$
\|x-\bar{x}\| \leq \frac{\rho L_{i}+l}{\rho \mu_{i}-\rho k_{i} L_{i}-k_{i} l} \min _{1 \leq i \leq m}\left\{\left\|h(x)-\Pi_{K(x)}^{f_{i}}\left[h(x)-\rho F_{i}(x)\right]\right\|\right\},
$$

which means that

$$
d(x, \operatorname{Sol}(\mathrm{VIMQVI})) \leq\|x-\bar{x}\| \leq \frac{\rho L_{i}+l}{\rho \mu_{i}-\rho k_{i} L_{i}-k_{i} l} r_{\rho}(x) .
$$

This completes the proof.

Remark 3.1 Lemma 2.2 implies that condition (3) holds under some suitable assumptions.

\section{Regularized gap functions and $D$-gap functions}

In general, the residual gap function fails to be smooth. For the algorithmic purpose, it is desirable to deal with a smooth optimization problem. Sun and Chai [32] and Charitha et al. [33] introduced the regularized gap function for GVVI and VVI, respectively. Li and Li [37] introduced the $D$-gap function for MSVI. Aussel et al. [31] constructed the $D$ gap function for IQVI. Taking motivation from these works, we design a regularized gap function and a $D$-gap function for VIMQVI and develop corresponding error bounds for VIMQVI. 


\subsection{Regularized gap function}

The regularized gap function for VIMQVI is defined for all $x \in R^{n}$ as follows:

$$
\phi_{\rho}(x)=\min _{1 \leq i \leq m} \sup _{y \in K(x)}\left\{\left\langle F_{i}(x), h(x)-y\right\rangle+f_{i}(h(x))-f_{i}(y)-\frac{1}{2 \rho}\|h(x)-y\|^{2}\right\},
$$

where $\rho>0$ is a parameter.

Lemma 4.1 We have

$$
\phi_{\rho}(x)=\min _{1 \leq i \leq m}\left\{\left\langle F_{i}(x), R_{\rho}^{i}(x)\right\rangle+f_{i}(h(x))-f_{i}\left(h(x)-R_{\rho}^{i}(x)\right)-\frac{1}{2 \rho}\left\|R_{\rho}^{i}(x)\right\|^{2}\right\},
$$

where

$$
R_{\rho}^{i}(x)=h(x)-\Pi_{K(x)}^{f_{i}}\left[h(x)-\rho F_{i}(x)\right], \quad \forall x \in R^{n} .
$$

And if $x \in h^{-1}(K)$, where $h^{-1}(K)=\left\{\xi \in R^{n} \mid h(\xi) \in K(\xi)\right\}$, then

$$
\phi_{\rho}(x) \geq \frac{1}{2 \rho} r_{\rho}(x)^{2}
$$

Proof For given $x \in R^{n}$ and $i \in\{1,2, \ldots, m\}$, set

$$
\psi_{i}(x, y)=\left\langle F_{i}(x), h(x)-y\right\rangle+f_{i}(h(x))-f_{i}(y)-\frac{1}{2 \rho}\|h(x)-y\|^{2}, \quad y \in R^{n} .
$$

Consider the following problem:

$$
g_{i}(x)=\max _{y \in K(x)} \psi_{i}(x, y)
$$

Since $\psi_{i}(x, \cdot)$ is a strongly concave function and $K(x)$ is nonempty closed and convex, the above optimization problem has a unique solution, say $z \in K(x)$. Invoking the optimality condition at $z$, we obtain

$$
0 \in F_{i}(x)+\partial f_{i}(z)+\frac{1}{\rho}(z-h(x))+N_{K(x)}(z)
$$

where $N_{K(x)}(z)$ is the normal cone at $z$ to $K(x)$ and $\partial f_{i}(z)$ denotes the subdifferential of $f_{i}$ at $z$. Therefore,

$$
\left\langle z-\left(h(x)-\rho F_{i}(x)\right), y-z\right\rangle+\rho f_{i}(y)-\rho f_{i}(z) \geq 0, \quad \forall y \in K(x)
$$

and so $z=\Pi_{K(x)}^{f_{i}}\left[h(x)-\rho F_{i}(x)\right]$. Hence $g_{i}(x)$ can be rewritten as

$$
\begin{aligned}
g_{i}(x)= & \left\langle F_{i}(x), h(x)-\Pi_{K(x)}^{f_{i}}\left[h(x)-\rho F_{i}(x)\right]\right\rangle \\
& +f_{i}(h(x))-f_{i}\left(\Pi_{K(x)}^{f_{i}}\left[h(x)-\rho F_{i}(x)\right]\right) \\
& -\frac{1}{2 \rho}\left\|h(x)-\Pi_{K(x)}^{f_{i}}\left[h(x)-\rho F_{i}(x)\right]\right\|^{2} .
\end{aligned}
$$


Letting $R_{\rho}^{i}(x)=h(x)-\Pi_{K(x)}^{f_{i}}\left[h(x)-\rho F_{i}(x)\right]$, we get

$$
g_{i}(x)=\left\langle F_{i}(x), R_{\rho}^{i}(x)\right\rangle+f_{i}(h(x))-f_{i}\left(h(x)-R_{\rho}^{i}(x)\right)-\frac{1}{2 \rho}\left\|R_{\rho}^{i}(x)\right\|^{2},
$$

and so

$$
\phi_{\rho}(x)=\min _{1 \leq i \leq m}\left\{\left\langle F_{i}(x), R_{\rho}^{i}(x)\right\rangle+f_{i}(h(x))-f_{i}\left(h(x)-R_{\rho}^{i}(x)\right)-\frac{1}{2 \rho}\left\|R_{\rho}^{i}(x)\right\|^{2}\right\} .
$$

From the definition of projection $\Pi_{K(x)}^{f_{i}}\left[h(x)-\rho F_{i}(x)\right]$, we have

$$
\begin{aligned}
& \left\langle\Pi_{K(x)}^{f_{i}}\left[h(x)-\rho F_{i}(x)\right]-h(x)+\rho F_{i}(x), y-\Pi_{K(x)}^{f_{i}}\left[h(x)-\rho F_{i}(x)\right]\right\rangle \\
& \quad+\rho f_{i}(y)-\rho f_{i}\left(\Pi_{K(x)}^{f_{i}}\left[h(x)-\rho F_{i}(x)\right]\right) \geq 0 .
\end{aligned}
$$

For any $x \in h^{-1}(K)$, we have $h(x) \in K(x)$, and therefore, by taking $y=h(x)$ in the above relation, we get

$$
\left\langle\rho F_{i}(x)-R_{\rho}^{i}(x), R_{\rho}^{i}(x)\right\rangle+\rho f_{i}(h(x))-\rho f_{i}\left(h(x)-R_{\rho}^{i}(x)\right) \geq 0,
$$

that is,

$$
\left\langle F_{i}(x), R_{\rho}^{i}(x)\right\rangle+f_{i}(h(x))-f_{i}\left(h(x)-R_{\rho}^{i}(x)\right) \geq \frac{1}{\rho}\left\langle R_{\rho}^{i}(x), R_{\rho}^{i}(x)\right\rangle=\frac{1}{\rho}\left\|R_{\rho}^{i}(x)\right\|^{2} .
$$

From the definition of $r_{\rho}(x)$ and (8), we get $\phi_{\rho}(x) \geq \frac{1}{2 \rho} r_{\rho}(x)^{2}$. This completes the proof. $\square$

Theorem 4.1 For $\rho>0$, $\phi_{\rho}$ is a gap function for VIMQVI on the $\operatorname{set}^{-1}(K)=\left\{\xi \in R^{n} \mid h(\xi) \in\right.$ $K(\xi)\}$.

Proof From the definition of $\phi_{\rho}$, we have

$$
\phi_{\rho}(x) \geq \min _{1 \leq i \leq m}\left\{\left\langle F_{i}(x), h(x)-y\right\rangle+f_{i}(h(x))-f_{i}(y)-\frac{1}{2 \rho}\|h(x)-y\|^{2}\right\}, \quad \forall y \in K(x) .
$$

Therefore, for any $x \in h^{-1}(K)$, by setting $y=h(x)$, we have $\phi_{\rho}(x) \geq 0$.

Suppose that $\bar{x} \in h^{-1}(K)$ with $\phi_{\rho}(\bar{x})=0$. From (9), it follows that $r_{\rho}(\bar{x})=0$, which implies that $\bar{x}$ is the solution of VIMQVI.

Conversely, if $\bar{x}$ is a solution of VIMQVI, there exists $1 \leq i_{0} \leq m$ such that

$$
\left\langle F_{i_{0}}(\bar{x}), h(\bar{x})-y\right\rangle+f_{i_{0}}(h(\bar{x}))-f_{i_{0}}(y) \leq 0, \quad \forall y \in K(\bar{x}),
$$

which means that

$$
\min _{1 \leq i \leq m}\left\{\sup _{y \in K(\bar{x})}\left\{\left\langle F_{i}(\bar{x}), h(\bar{x})-y\right\rangle+f_{i}(h(\bar{x}))-f_{i}(y)-\frac{1}{2 \rho}\|h(\bar{x})-y\|^{2}\right\}\right\} \leq 0 .
$$

Thus, $\phi_{\rho}(\bar{x}) \leq 0$. The previous assertion leads to $\phi_{\rho}(\bar{x}) \geq 0$ and it follows that $\phi_{\rho}(\bar{x})=0$. This completes the proof. 
Since, according to Theorem 4.1, $\phi_{\rho}$ can act as a gap function for VIMQVI, it is interesting to investigate the error bound properties that can be obtained with $\phi_{\rho}$. By Theorem 3.2 and (9), we obtain the following corollary directly.

Corollary 1 Let $F_{i}: R^{n} \rightarrow R^{n}(i=1,2, \ldots, m)$ be $L_{i}$-Lipschitz continuous, $h: R^{n} \rightarrow R^{n}$ be lLipschitz continuous, and for $i=1,2, \ldots, m,\left(F_{i}, h\right)$ be strongly monotone couples with modulus $\mu_{i}$. Let $\bigcap_{i=1}^{m} \operatorname{sol}(\mathrm{IMQVI})^{i} \neq \emptyset$. Assume that there exists $k_{i} \in\left(0, \frac{\mu_{i}}{L_{i}}\right)$ such that

$$
\left\|\Pi_{K(x)}^{f_{i}} z-\Pi_{K(y)}^{f_{i}} z\right\| \leq k_{i}\|x-y\|, \quad \forall x, y \in R^{n}, \forall z \in\left\{v \mid v=h(x)-\rho F_{i}(x)\right\} .
$$

Then, for any $x \in h^{-1}(K)$ and any $\rho>\frac{k_{i} l}{\mu_{i}-k_{i} L_{i}}$,

$$
d(x, \operatorname{Sol}(\mathrm{VIMQVI})) \leq \frac{\rho L_{i}+l}{\rho \mu_{i}-\rho k_{i} L_{i}-k_{i} l} \sqrt{2 \rho \phi_{\rho}(x)}
$$

\subsection{D-Gap functions}

It is remarkable that the regularized gap function $\phi_{\rho}$ fails to give global error bounds for VIMQVI on $R^{n}$. Solodov [26] proposed the $D$-gap function for MVI and obtained error bounds related to the $D$-gap function for MVI. Li and Li [37] introduced the D-gap function for MSVI and obtained error bounds. For more details, see [27-29, 31]. With this motivation we introduce the $D$-gap function for VIMQVI, which provides the global error bound for VIMQVI on $R^{n}$.

The $D$-gap function for VIMQVI with parameters $\alpha>\beta>0$ is defined as follows:

$$
\begin{aligned}
G_{\alpha \beta}(x)= & \min _{1 \leq i \leq m}\left\{\operatorname { s u p } _ { y \in K ( x ) } \left\{\left\langle F_{i}(x), h(x)-y\right\rangle+f_{i}(h(x))-f_{i}(y)\right.\right. \\
& \left.-\frac{1}{2 \alpha}\|h(x)-y\|^{2}\right\}-\sup _{y \in K(x)}\left\{\left\langle F_{i}(x), h(x)-y\right\rangle+f_{i}(h(x))\right. \\
& \left.\left.-f_{i}(y)-\frac{1}{2 \beta}\|h(x)-y\|^{2}\right\}\right\} .
\end{aligned}
$$

By (8) in Lemma 4.1, we know $G_{\alpha \beta}$ can be rewritten as

$$
\begin{aligned}
G_{\alpha \beta}(x)= & \min _{1 \leq i \leq m}\left\{\left\langle F_{i}(x), R_{\alpha}^{i}(x)\right\rangle+f_{i}(h(x))-f_{i}\left(h(x)-R_{\alpha}^{i}(x)\right)\right. \\
& -\frac{1}{2 \alpha}\left\|R_{\alpha}^{i}(x)\right\|^{2}-\left(\left\langle F_{i}(x), R_{\beta}^{i}(x)\right\rangle+f_{i}(h(x))-f_{i}\left(h(x)-R_{\beta}^{i}(x)\right)\right. \\
& \left.\left.-\frac{1}{2 \beta}\left\|R_{\beta}^{i}(x)\right\|^{2}\right)\right\},
\end{aligned}
$$

where $R_{\alpha}^{i}(x)=h(x)-\Pi_{K(x)}^{f_{i}}\left[h(x)-\alpha F_{i}(x)\right]$ and $R_{\beta}^{i}(x)=h(x)-\Pi_{K(x)}^{f_{i}}\left[h(x)-\beta F_{i}(x)\right], \forall x \in R^{n}$.

Theorem 4.2 For any $x \in R^{n}, \alpha>\beta>0$, we have

$$
\frac{1}{2}\left(\frac{1}{\beta}-\frac{1}{\alpha}\right) r_{\beta}^{2}(x) \leq G_{\alpha \beta}(x) \leq \frac{1}{2}\left(\frac{1}{\beta}-\frac{1}{\alpha}\right) r_{\alpha}^{2}(x) .
$$


Proof From the definition of $G_{\alpha \beta}(x)$, it follows that

$$
\begin{aligned}
G_{\alpha \beta}(x)= & \min _{1 \leq i \leq m}\left\{\left\langle F_{i}(x), R_{\alpha}^{i}(x)-R_{\beta}^{i}(x)\right\rangle-f_{i}\left(h(x)-R_{\alpha}^{i}(x)\right)\right. \\
& \left.-\frac{1}{2 \alpha}\left\|R_{\alpha}^{i}(x)\right\|^{2}+f_{i}\left(h(x)-R_{\beta}^{i}(x)\right)+\frac{1}{2 \beta}\left\|R_{\beta}^{i}(x)\right\|^{2}\right\} .
\end{aligned}
$$

For any given $i \in\{1,2, \ldots, m\}$, we set

$$
\begin{aligned}
g_{\alpha \beta}^{i}(x)= & \left\langle F_{i}(x), R_{\alpha}^{i}(x)-R_{\beta}^{i}(x)\right\rangle-f_{i}\left(h(x)-R_{\alpha}^{i}(x)\right)-\frac{1}{2 \alpha}\left\|R_{\alpha}^{i}(x)\right\|^{2} \\
& +f_{i}\left(h(x)-R_{\beta}^{i}(x)\right)+\frac{1}{2 \beta}\left\|R_{\beta}^{i}(x)\right\|^{2} .
\end{aligned}
$$

From $\Pi_{K(x)}^{f_{i}}\left[h(x)-\beta F_{i}(x)\right] \in K(x)$, by Lemma 2.1, we know

$$
\begin{aligned}
& \left\langle\Pi_{K(x)}^{f_{i}}\left[h(x)-\alpha F_{i}(x)\right]-\left(h(x)-\alpha F_{i}(x)\right), \Pi_{K(x)}^{f_{i}}\left[h(x)-\beta F_{i}(x)\right]\right. \\
& \left.\quad-\Pi_{K(x)}^{f_{i}}\left[h(x)-\alpha F_{i}(x)\right]\right\rangle \\
& \quad+\alpha f_{i}\left(\Pi_{K(x)}^{f_{i}}\left[h(x)-\beta F_{i}(x)\right]\right)-\alpha f_{i}\left(\Pi_{K(x)}^{f_{i}}\left[h(x)-\alpha F_{i}(x)\right]\right) \geq 0,
\end{aligned}
$$

which means that

$$
\begin{aligned}
& \left\langle\alpha F_{i}(x)-R_{\alpha}^{i}(x), R_{\alpha}^{i}(x)-R_{\beta}^{i}(x)\right\rangle \\
& \quad+\alpha f_{i}\left(h(x)-R_{\beta}^{i}(x)\right)-\alpha f_{i}\left(h(x)-R_{\alpha}^{i}(x)\right) \geq 0 .
\end{aligned}
$$

Combining (12) and (13), we get

$$
\begin{aligned}
g_{\alpha \beta}^{i}(x) & \geq \frac{1}{\alpha}\left\langle R_{\alpha}^{i}(x), R_{\alpha}^{i}(x)-R_{\beta}^{i}(x)\right\rangle-\frac{1}{2 \alpha}\left\|R_{\alpha}^{i}(x)\right\|^{2}+\frac{1}{2 \beta}\left\|R_{\beta}^{i}(x)\right\|^{2} \\
& =\frac{1}{2 \alpha}\left\|R_{\alpha}^{i}(x)-R_{\beta}^{i}(x)\right\|^{2}+\frac{1}{2}\left(\frac{1}{\beta}-\frac{1}{\alpha}\right)\left\|R_{\beta}^{i}(x)\right\|^{2} .
\end{aligned}
$$

Since $\Pi_{K(x)}^{f_{i}}\left[h(x)-\alpha F_{i}(x)\right] \in K(x)$, by Lemma 2.1, we have

$$
\begin{aligned}
& \left\langle\Pi_{K(x)}^{f_{i}}\left[h(x)-\beta F_{i}(x)\right]-\left(h(x)-\beta F_{i}(x)\right), \Pi_{K(x)}^{f_{i}}\left[h(x)-\alpha F_{i}(x)\right]\right. \\
& \left.\quad-\Pi_{K(x)}^{f_{i}}\left[h(x)-\beta F_{i}(x)\right]\right\rangle \\
& \quad+\beta f_{i}\left(\Pi_{K(x)}^{f_{i}}\left[h(x)-\alpha F_{i}(x)\right]\right)-\beta f_{i}\left(\Pi_{K(x)}^{f_{i}}\left[h(x)-\beta F_{i}(x)\right]\right) \geq 0 .
\end{aligned}
$$

Hence

$$
\left\langle\beta F_{i}(x)-R_{\beta}^{i}(x), R_{\beta}^{i}(x)-R_{\alpha}^{i}(x)\right\rangle+\beta f_{i}\left(h(x)-R_{\alpha}^{i}(x)\right)-\beta f_{i}\left(h(x)-R_{\beta}^{i}(x)\right) \geq 0,
$$

and so

$$
\begin{aligned}
\frac{1}{\beta}\left\langle R_{\beta}^{i}(x), R_{\alpha}^{i}(x)-R_{\beta}^{i}(x)\right\rangle \geq & \left\langle F_{i}(x), R_{\alpha}^{i}(x)-R_{\beta}^{i}(x)\right\rangle \\
& -f_{i}\left(h(x)-R_{\alpha}^{i}(x)\right)+f_{i}\left(h(x)-R_{\beta}^{i}(x)\right) .
\end{aligned}
$$


This and (12) imply that

$$
\begin{aligned}
g_{\alpha \beta}^{i}(x) & \leq \frac{1}{\beta}\left\langle R_{\beta}^{i}(x), R_{\alpha}^{i}(x)-R_{\beta}^{i}(x)\right\rangle-\frac{1}{2 \alpha}\left\|R_{\alpha}^{i}(x)\right\|^{2}+\frac{1}{2 \beta}\left\|R_{\beta}^{i}(x)\right\|^{2} \\
& =-\frac{1}{2 \beta}\left\|R_{\alpha}^{i}(x)-R_{\beta}^{i}(x)\right\|^{2}+\frac{1}{2}\left(\frac{1}{\beta}-\frac{1}{\alpha}\right)\left\|R_{\alpha}^{i}(x)\right\|^{2} .
\end{aligned}
$$

From (14) and (15), for any $i \in\{1,2, \ldots, m\}$, we obtain

$$
\frac{1}{2}\left(\frac{1}{\beta}-\frac{1}{\alpha}\right)\left\|R_{\beta}^{i}(x)\right\|^{2} \leq g_{\alpha \beta}^{i}(x) \leq \frac{1}{2}\left(\frac{1}{\beta}-\frac{1}{\alpha}\right)\left\|R_{\alpha}^{i}(x)\right\|^{2}
$$

Hence

$$
\frac{1}{2}\left(\frac{1}{\beta}-\frac{1}{\alpha}\right) \min _{1 \leq i \leq m}\left\{\left\|R_{\beta}^{i}(x)\right\|^{2}\right\} \leq \min _{1 \leq i \leq m}\left\{g_{\alpha \beta}^{i}(x)\right\} \leq \frac{1}{2}\left(\frac{1}{\beta}-\frac{1}{\alpha}\right) \min _{1 \leq i \leq m}\left\{\left\|R_{\alpha}^{i}(x)\right\|^{2}\right\}
$$

and so

$$
\frac{1}{2}\left(\frac{1}{\beta}-\frac{1}{\alpha}\right) r_{\beta}^{2}(x) \leq G_{\alpha \beta}(x) \leq \frac{1}{2}\left(\frac{1}{\beta}-\frac{1}{\alpha}\right) r_{\alpha}^{2}(x)
$$

This completes the proof.

Now we prove that $G_{\alpha \beta}$ is a global gap function for VIMQVI on the set $R^{n}$.

Theorem 4.3 For $0<\beta<\alpha, G_{\alpha \beta}$ is a gap function for VIMQVI on $R^{n}$.

Proof According to (11), we have $G_{\alpha \beta}(x) \geq 0, \forall x \in R^{n}$. Suppose that $\bar{x} \in R^{n}$ with $G_{\alpha \beta}(\bar{x})=0$, (11) implies that $r_{\beta}(\bar{x})=0$. By Theorem 3.1, we know $\bar{x}$ is a solution of VIMQVI.

Conversely, if $\bar{x}$ is a solution of VIMQVI, from Theorem 3.1, it follows that $r_{\alpha}(\bar{x})=0$. (11) means that $G_{\alpha \beta}(\bar{x})=0$. This completes the proof.

Immediately, by using Theorem 3.2 and (11), we obtain a global error bound for VIMQVI on the set $R^{n}$.

Corollary 2 Let $F_{i}: R^{n} \rightarrow R^{n}(i=1,2, \ldots, m)$ be $L_{i}$-Lipschitz continuous, $h: R^{n} \rightarrow R^{n}$ be lLipschitz continuous, and for $i=1,2, \ldots, m,\left(F_{i}, h\right)$ be strongly monotone couples with modulus $\mu_{i}$. Let $\bigcap_{i=1}^{m} \operatorname{sol}(\mathrm{IMQVI})^{i} \neq \emptyset$. Assume that there exists $k_{i} \in\left(0, \frac{\mu_{i}}{L_{i}}\right)$ such that

$$
\left\|\Pi_{K(x)}^{f_{i}} z-\Pi_{K(y)}^{f_{i}} z\right\| \leq k_{i}\|x-y\|, \quad \forall x, y \in R^{n}, z \in\left\{v \mid v=h(x)-\beta F_{i}(x)\right\} .
$$

Then, for any $x \in R^{n}$ and any $\beta>\frac{k_{i} l}{\mu_{i}-k_{i} L_{i}}$,

$$
d(x, \operatorname{Sol}(\mathrm{VIMQVI})) \leq \frac{\beta L_{i}+l}{\beta \mu_{i}-\beta k_{i} L_{i}-k_{i} l} \sqrt{\frac{2 \beta \alpha}{\alpha-\beta} G_{\alpha \beta}(x)}
$$




\section{Concluding remarks}

One of the classical approaches in the analysis of a variational inequality (VI) and its variants is to transform it into an equivalent optimization problem by the notion of gap functions. In addition, gap functions play a central role in deriving the so-called error bounds, which provide a measure of the distances between the solution set and an arbitrary feasible point. These motivate us to study and analyze different gap functions and error bounds for VIMQVI.

In this paper, we introduce a vector inverse mixed quasi-variational inequality (VIMQVI), which includes IVI, IMVI, IQVI, VVI, and GVVI as special cases. We propose three gap functions for the VIMQVI, i.e., the residual gap function, the regularized gap function, and the $D$-gap function, and obtain error bounds for VIMQVI under strong monotonicity and Lipschitz continuity of underlying mappings by using these gap functions. Our basic tool is the generalized $f$-projection operator, which is more general than the well-known proximal mapping, see [37]. If $i=1$ and $f_{1}(x)=0$ for all $x \in R^{n}$, then the results obtained in this paper collapse to the corresponding ones in [31] and [36].

Acknowledgements

The authors thank the anonymous referees for their careful reading and insightful suggestions.

Funding

This work is supported from the National Natural Science Foundation of China (11701479, 11526170, 11701478,

11771067) and Chinese Postdoctoral Science Foundation (2018M643434).

\section{Abbreviations}

Not applicable.

Availability of data and materials

Not applicable.

\section{Competing interests}

The authors declare that they have no competing interests.

Authors' contributions

All the authors contributed equally in the writing of this paper. They read and approved the final manuscript.

\section{Author details}

${ }^{1}$ Department of Mathematics, Southwest Jiaotong University, Chengdu, China. ${ }^{2}$ School of Mathematical Sciences, University of Electronic Science and Technology of China, Chengdu, China. ${ }^{3}$ Business School, Sichuan University, Chengdu, China.

\section{Publisher's Note}

Springer Nature remains neutral with regard to jurisdictional claims in published maps and institutional affiliations.

Received: 17 July 2019 Accepted: 14 August 2019 Published online: 01 September 2019

References

1. Ekeland, I., Temam, R.: Convex Analysis and Variational Problems. North-Holland, Amsterdam (1976)

2. Konnov, I.V., Volotskaya, E.O.: Mixed variational inequalities and economic equilibrium problems. J. Appl. Math. 2, 289-314 (2002)

3. Reddy, B.D.: Mixed variational inequalities arising in elastoplasticity. Nonlinear Anal. 19, 1071-1089 (1992)

4. He, B.S., Liu, H.X.: Inverse variational inequalities in the economic field: applications and algorithms. http://www.paper.edu.cn/releasepaper/content/200609-260

5. He, B.S., Liu, H.X., Li, M., He, X.Z.: PPA-based methods for monotone inverse variational inequalities. http://www.paper.edu.cn/releasepaper/content/200606-219

6. He, B.S., He, X.Z., Liu, H.X.: Solving a class of constrained black-box inverse variational inequalities. Eur. J. Oper. Res. 204, 391-401 (2010)

7. Li, X., Li, X.S., Huang, N.J.: A generalized $f$-projection algorithm for inverse mixed variational inequalities. Optim. Lett. 8, 1063-1076 (2014)

8. Giannessi, F.: Theorems of the alternative, quadratic programs and complementarity problems. In: Cottle, R.W., Giannessi, F., Lions, J.L. (eds.) Variational Inequalities and Complementarity Problems, pp. 151-186. Wiley, New York (1980) 
9. Yu, S.J., Yao, J.C.: On vector variational inequalities. J. Optim. Theory Appl. 89, 749-769 (1996)

10. Lee, G.M., Kim, D.S., Lee, B.S., Yen, N.D.: Vector variational inequality as a tool for studying vector optimization problems. Nonlinear Anal. 34, 745-765 (1998)

11. Giannessi, F.: Vector Variational Inequalities and Vector Equilibria: Mathematical Theories. Kluwer Academic, Dordrecht (2000)

12. Chen, G.Y., Huang, X.X., Yang, X.Q.: Vector Optimization: Set-Valued and Variational Analysis. Lecture Notes in Economics and Mathematical Systems. Springer, Berlin (2005)

13. Zeng, L.C., Yao, J.C.: Existence of solutions of generalized vector variational inequalities in reflexive Banach spaces. J. Glob. Optim. 36, 483-497 (2006)

14. Yang, X.Q., Yao, J.C.: Gap functions and existence of solutions to set-valued vector variational inequalities. J. Optim. Theory Appl. 115, 407-417 (2002)

15. Chen, G.Y., Goh, C.J., Yang, X.Q.: On gap functions for vector variational inequalities. In: Giannessi, F. (ed.) Vector Variational Inequality and Vector Equilibria: Mathematical Theories, pp. 55-70. Kluwer Academic, Boston (2000)

16. Chen, Z:: Asymptotic analysis for proximal-type methods in vector variational inequality problems. Oper. Res. Lett. 43(3), 226-230 (2015)

17. Luu, D.V., Mai, T.T.: Optimality conditions for Henig efficient and superefficient solutions of vector equilibrium problems. J. Nonlinear Funct. Anal. 2018, Article ID 18 (2018)

18. Kim, J.K., Khanna, A.K., Ram, T.: On $\eta$-generalized operator variational-like inequalities. Commun. Optim. Theory 2018, Article ID 14 (2018)

19. Chen, J.W., Kobis, E.M., Kobis, A., Yao, J.C.: Optimality conditions for solutions of constrained inverse vector variational inequalities by means of nonlinear scalarization. J. Nonlinear Var. Anal. 1, 145-158 (2017)

20. Li, S.J., Chen, G.Y.: Properties of gap function for vector variational inequality. In: Giannessi, F., Maugeri, A. (eds.) Variational Analysis and Applications, pp. 605-631. Springer, Berlin (2005)

21. Li, S.J., Yan, H., Chen, G.Y.: Differential and sensitivity properties of gap functions for vector variational inequalities. Math. Methods Oper. Res. 57, 377-391 (2003)

22. Meng, K.W., Li, S.J.: Differential and sensitivity properties of gap functions for Minty vector variational inequalities. J. Math. Anal. Appl. 337, 386-398 (2008)

23. Li, S.J., Teo, K.L., Yang, X.Q., Wu, S.Y.: Gap functions and existence of solutions to generalized vector quasi-equilibrium problems. J. Glob. Optim. 34, 427-440 (2006)

24. Huang, N.J., Li, J., Yao, J.C.: Gap functions and existence of solutions for a system of vector equilibrium problems. J. Optim. Theory Appl. 133, 201-212 (2007)

25. Charitha, C., Dutta, J.: Regularized gap functions and error bounds for vector variational inequality. Pac. J. Optim. 6 497-510 (2010)

26. Solodov, M.V.: Merit functions and error bounds for generalized variational inequalities. J. Math. Anal. Appl. 287, 405-414 (2003)

27. Gupta, R., Mehra, A.: Gap functions and error bounds for quasi variational inequalities. J. Glob. Optim. 53, 737-748 (2012)

28. Pappalardo, M., Mastroeni, G., Passacantando, M.: Merit functions: a bridge between optimization and equilibria. Ann. Oper. Res. 240, 271-299 (2016)

29. Aussel, D., Correa, R., Marechal, M.: Gap functions for quasi-variational inequalities and generalized Nash equilibrium problems. J. Optim. Theory Appl. 151, 474-488 (2011)

30. Facchinei, F., Pang, J.S.: Finite-Dimensional Variational Inequalities and Complementarity Problems. Springer, Berlin (2003)

31. Aussel, D., Gupta, R., Mehra, A.: Gap functions and error bounds for inverse quasi-variational inequality problems. J. Math. Anal. Appl. 407, 270-280 (2013)

32. Sun, X.K., Chai, Y.: Gap functions and error bounds for generalized vector variational inequalities. Optim. Lett. 8, $1663-1673(2014)$

33. Charitha, C., Dutta, J., Lalitha, C.S.: Gap functions for vector variational inequalities. Optimization 64(7), 1499-1520 (2015)

34. Wu, K.Q., Huang, N.J.: The generalized $f$-projection operator with an application. Bull. Aust. Math. Soc. 73, 307-317 (2006)

35. Fan, J.H., Liu, X., Li, J.L.: Iterative schemes for approximating solutions of generalized variational inequalities in Banach spaces. Nonlinear Anal. 70, 3997-4007 (2009)

36. Li, X., Zou, Y.Z.: Existence result and error bounds for a new class of inverse mixed quasi-variational inequalities. J. Inequal. Appl. 2016, Article ID 42 (2016)

37. Li, C.Q., Li, J.: Merit functions and error bounds for constrained mixed set-valued variational inequalities via generalized $f$-projection operators. Optimization 65(8), 1569-1584 (2016) 